Bond University

Research Repository

\title{
Assessing lower-body peak power in elite rugby-union players
}

Argus, Christos K; Gill, Nicholas D; Keogh, Justin W L; Hopkins, Will G

Published in:

Journal of Strength and Conditioning Research

DOI:

10.1519/JSC.0b013e3181ddfabc

Licence:

Other

Link to output in Bond University research repository.

Recommended citation(APA):

Argus, C. K., Gill, N. D., Keogh, J. W. L., \& Hopkins, W. G. (2011). Assessing lower-body peak power in elite rugby-union players. Journal of Strength and Conditioning Research, 25(6), 1616-21.

https://doi.org/10.1519/JSC.0b013e3181ddfabc

\section{General rights}

Copyright and moral rights for the publications made accessible in the public portal are retained by the authors and/or other copyright owners and it is a condition of accessing publications that users recognise and abide by the legal requirements associated with these rights.

For more information, or if you believe that this document breaches copyright, please contact the Bond University research repository coordinator. 
Title: Assessing lower-body peak power in elite rugby union players. JSCR-08-371. Revision 2.

Running head: Assessing lower-body power.

Authors: Christos K. Argus, Nicholas D. Gill, Justin W. L. Keogh, Will G. Hopkins. Institute of Sport and Recreation Research New Zealand, AUT University, Auckland NZ.

\section{Corresponding author:}

Christos Argus

Institute of Sport and Recreation Research New Zealand, Faculty of Health and Environmental

Sciences, Auckland University of Technology, Private Bag 92006, Auckland 1020, New

Zealand.

Email: argy15@hotmail.com

Phone: +64 212419159

Facsimile: +64 92358885

Title: Assessing lower-body peak power in elite rugby union players. 
JSCR-08-371 Assessing lower-body power. 2

Running head: Assessing lower-body power. 


\begin{abstract}
Resistance training at the load that maximizes peak power (Pmax) may produce greater increases in peak power than other loads. Pmax for lower-body lifts can occur with no loading, but whether Pmax can be increased further with negative loading is unclear. The purpose of this investigation was therefore to determine lower-body Pmax (jump squat) using a spectrum of loads reps? . Box squat 1RM was measured in 26 elite rugby-union players. Pmax was then determined using loads of -28 to 60 \%1RM. Elastic bands were used to unload body weight for negative loads. Jump-squat Pmax occurred with no loading (bodyweight) in all but two subjects. There was a discontinuity in the power-load relationship for the jump squat, possibly due to the increased counter-movement in the bodyweight jump. These findings highlight methodological issues that need to be taken into consideration when comparing power outputs of loaded and unloaded jumps.
\end{abstract}

Key words: ELITE ATHLETES, JUMP SQUAT, POWER PROFILE. 


\section{INTRODUCTION}

Rugby union is a competitive sport which requires high levels of muscular power. As such, training methods that enhance muscular power are of extreme importance for the physical preparation in these athletes. The load that maximizes peak power (Pmax) has been discussed for more than 20 years and has been suggested to enhance power and performance in explosive exercise $(4,19,25)$. It has been proposed that training at the load that maximizes power may provide favourable neural and muscular adaptations $(19,25,29)$.

To accurately determine the effects of training at Pmax, Pmax must be firstly identified. However, large variations in the load that produces Pmax have been reported (2-4, 8, 10, 15, 16, 19). Traditionally, findings suggest that Pmax is typically expressed at loads ranging from $30 \%$ to $70 \%$ of maximum strength $(3,4,16)$. More recently, some studies have reported that Pmax occurs at loads less than $30 \%$ of maximal strength $(8,10)$. The large between-study variation in Pmax appears to be due to differences in exercise performed, methods used to assess power, and participants recruited (12). As such, using a Pmax load from the literature in the overall power training program of your athletes may not match their Pmax load, thereby producing a suboptimal loading and response.

To accurately quantify Pmax, power outputs across multiple loads need to be investigated. Recently, researchers have reported that vertical jump Pmax occurred when using bodyweight only $(8,10)$. However, power was not assessed at loads less than bodyweight. As such, whether Pmax can be increased further with negative loading is unclear. A novel approach to assess 
bodyweight at negative loads is with the use of elastic bands that may be attached in a manner which provides upward tension, thereby reducing the effective bodyweight of the subject.

Another methodological issue is that many investigations have assessed Pmax using single efforts (repetitions) at each load $(8,9,11,15,16)$, whereas power training typically consists of performing sets of three to five consecutive repetitions (5). Furthermore, recent literature has revealed that power is not maximised until the second or third repetition of a set (6). If the overall aim is to train at Pmax (using consecutive repetitions); then Pmax should be assessed in the same manner. To date only Baker and colleagues (2-4) have assessed Pmax in a training environment performing multiple consecutive repetitions and reported that Pmax occurred between $40-70 \%$ of maximum strength. Finally, the experience level (or training history) of subjects assessed may produce variation in the findings. Baker (2) reported that stronger athletes may produce Pmax at lower intensities than weaker athletes. Therefore to make accurate comparison between investigations, subjects need to be of similar strength levels.

Elite rugby union athletes typically have high levels of strength and regularly perform resistance training with multiple sets and consecutive repetitions. If methodology issues have an effect on the load that maximises peak power, then specific population assessment needs to occur to accurately identify Pmax. Determining Pmax in this population will provide athletes with specific training intensities that allow maximal peak power to be achieved during training, which in turn may lead to enhanced performance gains $(19,25,29)$. 
Therefore, the purpose of this investigation was to determine lower-body Pmax in elite rugby union players. Points of difference from most if not all previous investigations included the assessment of Pmax loads at negative through to positive loads and the performance of multiple consecutive repetitions at each load.

\section{METHODS}

\section{Experimental Approach to the Problem do you still need refs to bench (ref 3 and 5 I}

\section{think?)}

In order to more accurately quantify Pmax in terms of how it is commonly applied to training programs, elite rugby union subjects were assessed for lower-body maximal strength and power (via a spectrum of loads including negative loading) across four separate sessions, with each session separated by 24 hours (Table 1). Multiple repetitions were performed in each set (one set of four repetitions at each load) to be more representative of a typical training session. Peak power was selected as the dependent measure as it has been reported to have the greatest association with athletic performance (14). Power was assessed using the jump squat exercise due to its common usage in power training programs and research studies and its ability to represent lower-body power $(1,4)$.

Insert Table 1 about here

\section{Subjects}

Eighteen elite rugby union players from a Super 14 professional rugby team during the preseason phase of their campaign volunteered to take part in this study (mean \pm SD; age, $23.8 \pm 2.2$ 
years; height, $185.8 \pm 6 \mathrm{~cm}$; mass, $103.8 \pm 10.6 \mathrm{~kg}$ ). Each subject had undergone at least two years of intensive and regular resistance training exercise, and must have been competing in a prior national or international rugby competition to be included in this study. Subjects were informed of the experimental risks and signed an informed consent document prior to the investigation. The investigation was approved by an Institutional Review Board for use of Human subjects (Auckland University of Technology Ethics Committee). Four subjects were unable to attend session two due to unforeseen circumstances.

\section{Procedures}

\section{Strength}

Maximal strength was assessed using the box squat exercise using methods previously outlined (1). Briefly, following three sub-maximal sets of box squat, each athlete then performed one set to failure of one to four repetitions. Participants used a self-selected foot position and were required to lower themselves to a sitting position briefly on the box and then return to a standing position. The box height was adjusted for each athlete to allow the top of the thighs to be parallel to the floor while in the seated position. The box squat was performed using free weights. Three minutes rest was allowed between each set. Each set to failure was used to predict the athletes' one repetition maximum (1RM).

The following equation was used to predict box squat 1RM (21). This equation is a valid measure of $1 \mathrm{RM}$ strength as it has been show to have a correlation between actual and predicted 1RM of $r=0.969$ (22):

$$
1 \mathrm{RM}=(100 \mathrm{x} \text { weight }) /(101.3-(2.67123 \times \text { reps }))
$$




\section{Jump Squat}

Lower-body power was assessed using a jump squat exercise performed in a Smith machine. Subjects warmed up with two sets of four repetitions lowering the bar to a $90^{\circ}$ knee angle using a load of $50 \%$ of their 1RM box squat. Subjects then completed one set of four repetitions of jump squats at $-28 \%$ ( $\pm 5 \%$ ), $-15 \%$ ( $\pm 3 \%$ ), $0 \%$ (bodyweight), $20 \%, 30 \%, 40 \%, 50 \%$ or $60 \% 1 \mathrm{RM}$ box squat. Subjects used a self selected foot position and lowered the bar to a self selected depth during these performance tests. Subjects were then required to jump as explosively as possible trying to jump as high as they could (1). Three minutes rest was allowed between each set. The bodyweight jump was assessed using a broomstick which was placed behind the neck and on the top of the shoulders. The $-28 \%( \pm 5 \%)$ and $-15 \%( \pm 3 \%)$ jump squats were an assisted jump, performed in a squat cage wearing a climber's harness with an elastic band (Iron Woody LLC, Olney MT, USA) attached to either side of the harness (at the hip level), with the other end attached above the participant to the top of the squat cage. Two thicknesses of elastic bands were used. The elastic bands provided vertical tension which reduced the body weight of each participant when the participant was in a standing position with hip and knee fully extended. The reduction in weight was assessed by having subjects stand on scales with and without the attachment of the elastic bands.

The power and displacement produced during each repetition was quantified with a Gymaware ${ }^{\mathrm{TM}}$ optical encoder (50 Hz sample period with no data smoothing or filtering; Kinetic Performance Technology, Canberra, Australia) using the methods described elsewhere (13). Quantification of the power produced included bodyweight and bar mass (system mass) in the calculation (14). 


\section{Statistical Analyses}

To estimate the load that maximized mechanical power output, a quadratic was fitted to each participant's power output (in Watts) and load (\% of 1RM). However, in all but two subjects, power at bodyweight was clearly above any quadratic curve fitted to the points (Figure 1). Additionally, for the four subjects that did not complete the assisted jumps, the quadratic curves all had positive curvature where theory predicts negative curvature. Therefore for all subjects we used the value observed at bodyweight for Pmax. Findings were discussed as means and standard deviations.

Insert Figure 1 about here

In addition to fitting a quadratic, standardised differences of the mean were used to assess magnitudes of effects between each individual load assessed by dividing the differences by the appropriate between-athlete standard deviation. Standardised changes of $<0.20,<0.60$, $<1.2,<2.0$ and $>2.0$ were interpreted as trivial, small, moderate, large and very large effects, respectively $(7,18)$. Lastly, displacement data were log-transformed to reduce non-uniformity of error, and the differences were derived by back transformation as percent changes (17). To make inferences about the true (large-sample) value of an effect, the uncertainty in the effects were expressed as $90 \%$ confidence limits.

The interclass correlation (ICC) and coefficient of variation (CV) for box squat was $\mathrm{r}=0.915$ and 4.6\%, respectively. The ICC's and CV\% for jump squat at $0 \%$ and $50 \%$ of $1 \mathrm{RM}$ box squat were 
0.834 and $4.2 \%$, and 0.904 and $4.8 \%$, respectively. All test-retest reliabilities were assessed seven days apart. Validity of the Gymaware ${ }^{\mathrm{TM}}$ optical encoder has been previously reported elsewhere (13). The sample size for this investigation was limited to the number of athletes in the squad. All athletes in the squad that were injury free were included and therefore no more athletes could be obtained.

\section{RESULTS}

The mean predicted $1 \mathrm{RM}$ box squat was $147.9 \mathrm{~kg}$ ( $\pm 26.8 \mathrm{~kg})$. The greatest lower-body peak power was $8880 \mathrm{~W}( \pm 2186 \mathrm{~W})$ and occurred at bodyweight (Figure 2). The peak power produced during the bodyweight jump was greater (moderate to large effect size) than that of all other intensities assessed. Sixteen out of the 18 subjects produced peak lower-body power at bodyweight (Figure 4). Due to the irregularity in the lower-body power results, whereby a quadratic could not be fitted to the points (see Statistical Analyses; Figure 1); we re-examined the GymAware ${ }^{\mathrm{TM}}$ data to gain some insight into the potential reasons underlying this result. As the GymAware ${ }^{\mathrm{TM}}$ system is a linear position transducer, we started by examining the displacement data to ascertain whether differences in technique between the different jump intensities may have contributed to this finding.

Analysis of the displacement data revealed that during the bodyweight jump, the self selected depth (dip) prior to the propulsive phase of the jump was greater by $24 \pm 11 \%$ to $40 \pm 16 \%$ (moderate to large effect size) than all positive loads. As the loads increased, the subjects continued to reduce the depth of their countermovement. Small differences in the countermovement depth ranging from $11 \%( \pm 11 \%)$ to $17 \%$ ( $\pm 14 \%)$ were observed between $20 \%$ 
and $40 \%$, 20\% and 50\%, and 20\% and 60\% 1RM box squat load. Additionally, small differences ranging from $7 \%( \pm 9 \%)$ to $14 \%( \pm 9 \%)$ were also observed between $30 \%$ and $50 \%$, $30 \%$ and $60 \%$, and $40 \%$ and $60 \%$ 1RM box squat load.

Insert Figure 2 about here

\section{DISCUSSION}

It has been hypothesized that training at Pmax is beneficial for increasing muscular power $(4,19$, 25). Therefore the purpose of this investigation was to determine lower-body Pmax in elite rugby union players. To the author's knowledge, this was the first investigation that assessed Pmax using negative loads. By assessing power at negative loading we were able to identify a decline in power either side of the maximum power output which previous authors have not considered $(8,10)$. Peak lower-body power occurred with no loading (bodyweight) in all but two subjects. However, discontinuity in the power outputs of the lower-body was observed between bodyweight and all loaded jumps.

An interesting phenomenon occurred when assessing lower-body power across this spectrum of negative and positive loads. In all but two of the subjects assessed, power with no loading was substantially higher than all other loads assessed and was clearly above any quadratic curve that was fitted to all the points (Figure 1). On closer observation there appeared to be discontinuity of the power outputs between bodyweight and all positive loads. Indeed negative and bodyweight loads appeared to have a different power-load relationship than the positive loads. As such, it may be that a separate quadratic needs to be fitted to each power-load relationship when loaded 
and unloaded intensities are assessed. However, this would result in two Pmax intensities, one for training with unloaded jumps and the other for loaded jumps.

The separate power-load relationships may suggest that something substantially affects power output when subjects jump with an additional load. We re-analysed the position data produced by Gymaware ${ }^{\mathrm{TM}}$ and found that during the bodyweight jump, the self selected depth (dip) prior to the propulsive phase of the jump was greater than all loaded jumps (24\% - 40\%). Furthermore, as the loads increased to a greater percent of $1 \mathrm{RM}$ there was a further reduction in the depth of the counter-movement. As such, the disproportionally higher power output at bodyweight may be due to the larger dip used in this jump. The use of a greater dip with the bodyweight load may have afforded this jump some biomechanical advantages that contributed to the greater power outputs. The deeper countermovement would have increased the time to produce force. According to the impulse-momentum relationship, greater time to produce force would increase the amount of impulse (force multiplied by time) generated, which in turn would result in a greater change in the momentum (velocity) of the system (20). Additionally, the greater dip would have increased the amount of stretch placed on the agonist musculature, and via the forcelength relationship allow greater forces to be generated (26).

The methodological concerns observed could be controlled by keeping the depth consistent for all jumps. However, what should the constant depth be? If it is too low, velocity of the movement may be compromised and there is chance of increasing the likelihood of injury when jumping with heavy loads. If it is not low enough, it may prevent an optimal combination of force and velocity reducing power output and defeating the purpose of assessing Pmax. 
Additionally, how should depth be controlled? Cormie and colleagues (10) attempted to control depth by visually monitoring knee angle to a depth of $90^{\circ}$. However, Cormie and colleagues (10) still reported significant differences in depth between the different loading intensities. Harris and colleagues (15) controlled depth by performing a concentric only jump squat starting at a fixed knee angle of $110^{\circ}$. However, what if the purpose of your training was to improve stretch shortening cycle and countermovement peak power? Young and colleagues (30) suggested that executing a countermovement at a self selected depth encouraged subjects to find their own optimum jumping conditions. Furthermore, as previously alluded to in the introduction, if the goal is to train at the Pmax load, Pmax needs to be assessed in the manner it is trained. For most athletes, they will train using a self selected depth.

The discontinuity in jump technique (amount of dip) between each load makes determining Pmax for the lower-body problematic. If lower-body Pmax can not be accurately determined; then the contention that training at the load that maximises power may provide favourable neural and muscular adaptations $(19,25,29)$ would appear somewhat problematic, at least for the lower-body.

Lower-body peak power occurred at bodyweight, a finding similar to Cormie and colleagues (8) who reported that lower-body peak power occurred at bodyweight in well trained (football players, long jumpers and sprinters) (8) and untrained males (10). In contrast, Siegel and colleagues (27) reported that peak power occurred between $50 \%$ and $70 \% 1 \mathrm{RM}$ squat in untrained subjects, while Sleivert and Tainghue (28) reported that peak power occurred at $60 \%$ of $1 \mathrm{RM}$ squat in trained athletes. The difference in findings is likely due to the inclusion or 
exclusion of system mass (i.e. bar mass plus bodyweight) in the calculation of power. In the current investigation, and investigations by Cormie and colleagues $(8,10)$; all of which found peak power to occur at bodyweight, system mass was included in the calculation of power. Whereas the investigation by Sleivert and Taingahue (28) used bar mass only. Additionally, Siegel and colleagues (27) did not state that system mass was included in their calculations. This becomes extremely important when comparing findings as the inclusion or exclusion of bodyweight can cause a shift in peak power from 20\% (system mass included) to $70 \%$ of 1RM (system mass excluded) (14). Therefore the higher Pmax observed in the two investigations may be artificially high due to the exclusion of system mass from the calculation.

Heavy strength training and/or high velocity training has been shown to be effective in improving explosive performance in some studies $(19,23,24)$. However, it has been suggested that training at Pmax may enhance power and performance in explosive exercise more so than heavy strength and/or high velocity training $(4,19,25)$. It should be noted that there is only a limited and equivocal literature involving the comparisons of training at Pmax vs. heavier and/or lighter loads, and as such the load that maximises performance adaptation is still somewhat unknown.

The load that maximizes peak power may be influenced by several factors including the spectrum of loads assessed and whether comparisons are made between loaded and unloaded conditions. Additionally, data calculation and reporting methods (i.e. inclusion or exclusion of bodyweight) may influence Pmax. 


\section{PRACTICAL APPLICATIONS}

Lower-body Pmax occurred at bodyweight in all but two subjects, however results indicated there was a discontinuity between loaded and unloaded jumps. As such lower-body current Pmax assessment procedures may be flawed due to the inability to accurately determine the load that maximises peak power. Methods that can assess and improve lower-body power in a training environment need to be developed. We suggest assessment using a range of heavy and lighter intensities for each individual in each exercise, in a manner similar to how he or she trains. This will increase external validity and possibly result in a greater likelihood of enhanced peak power or training adaptations.

\section{REFERENCES}

1. ARGUS, CK, GILL, ND, KEOGH, JWL, HOPKINS, WG, and BEAVEN, CM. Changes in strength, power and steroid hormones during a professional rugby union competition. $J$ Strength Cond Res 23:1583-1592. 2009.

2. BAKER, D. A series of studies on the training of high-intensity muscle power in rugby league football players. J Strength Cond Res 15:198-209. 2001.

3. BAKER, D, NANCE, S, and MOORE, M. The load that maximizes the average mechanical power output during explosive bench press throws in highly trained athletes. J Strength Cond Res 15:20-24. 2001.

4. BAKER, D, NANCE, S, and MOORE, M. The load that maximizes the average mechanical power output during jump squats in power-trained athletes. $J$ Strength Cond Res 15:92-97. 2001.

5. BAKER, D and NEWTON, RU. Methods to increase the effectiveness of maximal power training for the upper body. Strength Cond J 27:24. 2005.

6. BAKER, DG and NEWTON, RU. Change in power output across a high-repetition set of bench throws and jump squats in highly trained athletes. J Strength Cond Res 21:10071011. 2007.

7. BATTERHAM, AM and HOPKINS, WG. Making meaningful inferences about magnitudes. Int J Sports Physiol Perfom 1:50-57. 2006.

8. CORMIE, P, GRANT, OM, TRIPLETT, NT, and MCBRIDE, JM. Optimal loading for maximal power output during lower-body resistance exercises. Med Sci Sport Exercise 39:340-349. 2007.

9. CORMIE, P, MCBRIDE, JM, and MCCAULLEY, GO. The influence of body mass on calculation of power during lower-body resistance exercises. J Strength Cond Res 21:1042-1049. 2007. 
10. CORMIE, P, MCBRIDE, JM, and MCCAULLEY, GO. Power-time, force-time, and velocity-time curve analysis during the jump squat: Impact of load. J Appl Biomech 24:112-120. 2008.

11. CRONIN, J, MCNAIR, PJ, and MARSHALL, RN. Developing explosive power: a comparison of technique and training. J Sci Med Sport 4:59-70. 2001.

12. CRONIN, J and SLEIVERT, G. Challenges in understanding the influence of maximal power training on improving athletic performance. Sport Med 35:213-234. 2005.

13. DRINKWATER, EJ, GALNA, B, MCKENNA, MJ, HUNT, PH, and PYNE, DB. Validation of an optical encoder during free weight resistance movements and analysis of bench press sticking point power during fatigue. J Strength Cond Res 21:510-517. 2007.

14. DUGAN, EL, DOYLE, TLA, HUMPHRIES, B, HASSON, CJ, and NEWTON, RU. Determining the optimal load for jump squats: A review of methods and calculations. $J$ Strength Cond Res 18:668-674. 2004.

15. HARRIS, NK, CRONIN, JB, and HOPKINS, WG. Power outputs of a machine squatjump across a spectrum of loads. J Strength Cond Res 21:1260-1264. 2007.

16. HARRIS, NK, CRONIN, JB, HOPKINS, WG, and HANSEN, KT. Squat jump training at maximal power loads vs. heavy loads: effect on sprint ability. J Strength Cond Res 22:1742-1749. 2008.

17. HOPKINS, WG. Spreadsheets for analysis of controlled trials with adjustment for a predictor. Sportscience 10 (sportsci.org/2006/wghcontrial.htm) 2006.

18. HOPKINS, WG, MARSHALL, SW, BATTERHAM, AM, and HANIN, J. Progressive statistics for studies in sports medicine and exercise science. Med Sci Sport Exercise 41:3-12. 2009.

19. KANEKO, M, FUCHIMOTO, T, TOJI, H, and SUEI, K. Training effect of different loads on the force-velocity relationship and mechanical power output in human muscle. Scand J Sports Sci 5:50-55. 1983.

20. KREIGHBAUM, E and BARTHELS, K, eds. Linear momentum and kinetic energy. Biomechanics. A qualitative approach for studying human movement, ed. E. Kreighbaum and K. Barthels. 1996, Allyn and Bacon: Needhham Heights, MA. 290-299.

21. LANDER, J. Maximums based on reps. Natl Str Cond Assoc 6:60-61. 1985.

22. LESUER, DA, MCCORMICK, JH, MAYHEW, JL, WASSERSTEIN, RL, and ARNOLD, MD. The accuracy of prediction equations for estimating 1-RM performance in the bench press, squat, and deadlift. J Strength Cond Res 11:211-213. 1997.

23. MANGINE, GT, RATAMESS, NA, HOFFMAN, JR, FAIGENBAUM, AD, KANG, J, and CHILAKOS, A. The effects of combined ballistic and heavy resistance training on maximal lower- and upper-body strength in recreationally trained men. J Strength Cond Res 22:132-139. 2008.

24. MCBRIDE, JM, TRIPLETT-MCBRIDE, T, DAVIE, A, and NEWTON, RU. The effect of heavy- vs. light-load jump squats on the development of strength, power, and speed. $J$ Strength Cond Res 16:75-82. 2002.

25. NEWTON, RU and KRAEMER, WJ. Developing explosive muscular power: Implications for a mixed methods training strategy. Strength Cond 16:20-31. 1994.

26. SCHACHAR, R, HERZOG, W, and LEONARD, TR. Force enhancement above the initial isometric force on the descending limb of the force-length relationship. $J$ Biomech 35:1299. 2002. 
27. SIEGEL, JA, GILDERS, RM, STARON, RS, and HAGERMAN, FC. Human muscle power output during upper- and lower-body exercises. J Strength Cond Res 16:173-178. 2002.

28. SLEIVERT, G and TAINGAHUE, M. The relationship between maximal jump-squat power and sprint acceleration in athletes. Eur J Appl Physiol 91:46-52. 2004.

29. WILSON, GJ, NEWTON, RU, MURPHY, AJ, and HUMPHRIES, BJ. The optimal training load for the development of dynamic athletic performance. Med Sci Sport Exercise 25:1279-1286. 1993.

30. YOUNG, WB, JENNER, A, and GRIFFITHS, K. Acute enhancement of power performance from heavy load squats. J Strength Cond Res 12:82-84. 1998.

\section{ACKNOWLEDGEMENTS}

The Waikato Rugby Union and the Tertiary Education Commission provided finical support by the way of scholarship for the primary author. The results of the present study do not constitute endorsement by the NSCA. 


\section{Figure Legends}

Figure 1. Examples from three different subjects of quadratics fitted to power outputs at different intensities. The data point on the vertical axis represents bodyweight. A, example of best fit. B, example of typical fit. C, example of worst fit.

Figure 2. Jump squat peak power at a spectrum of intensities in elite rugby union players. RM, Repetition maximum. $\mathrm{n}=20$. \#, denotes moderate to large differences (effect size) between $0 \%$ and all other loads. 


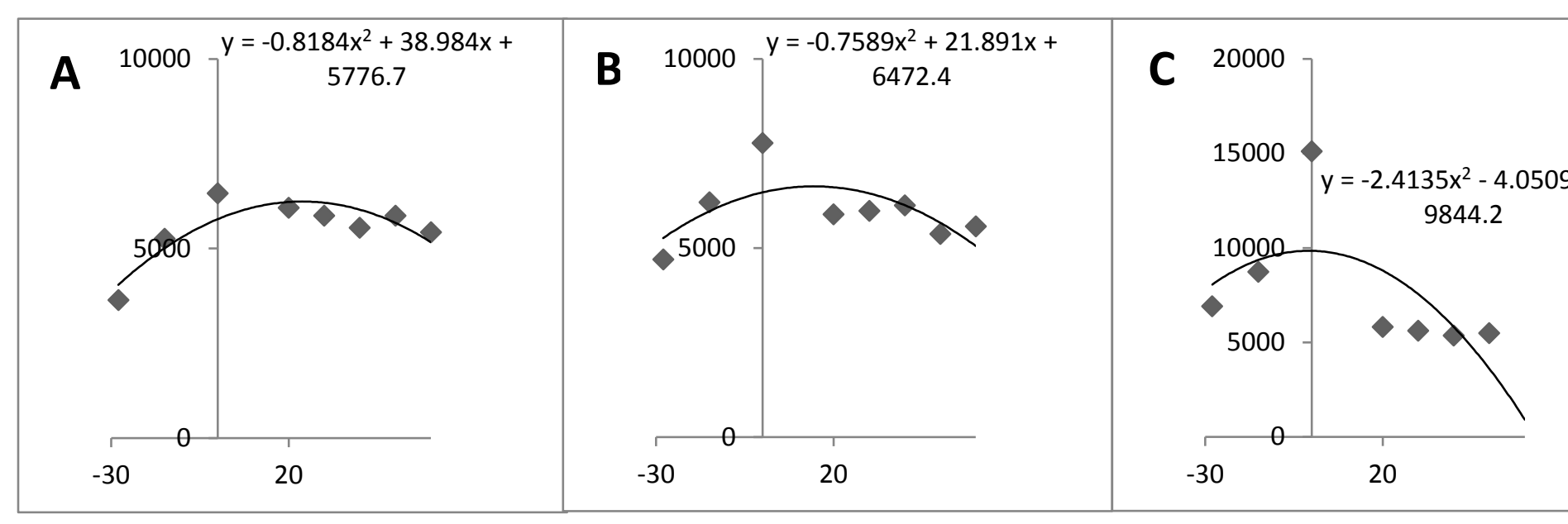

Figure 1. Examples from three different subjects of quadratics fitted to power outputs at different intensities.

The data point on the vertical axis represents bodyweight. A, example of best fit. B, example of typical fit. C, example of worst fit. 


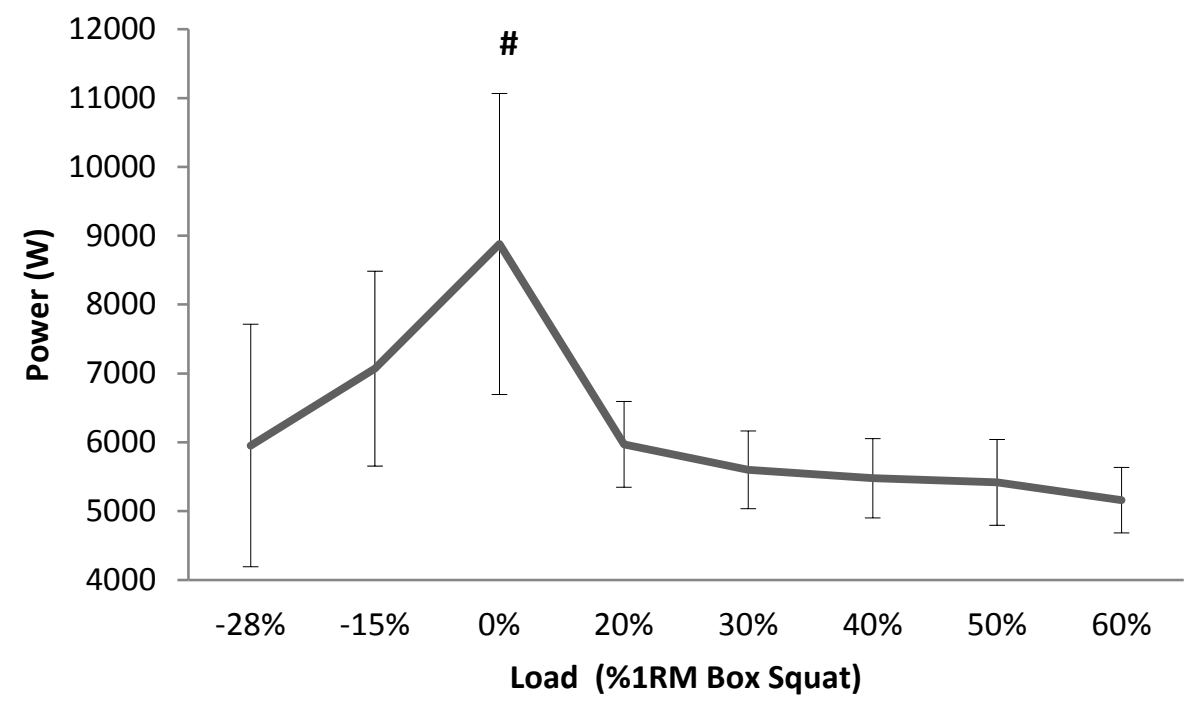

Figure 2. Jump squat peak power at a spectrum of intensities in elite rugby union players. RM, Repetition maximum. $\mathbf{n}=20$. \#, denotes moderate to large differences (effect size) between $0 \%$ and all other loads. 
JSCR-08-371 Assessing lower-body power. 21

Table 1. Order and outline of strength and power assessments.

\begin{tabular}{cccc} 
Order & Mode & Exercise & Load \\
\hline Session One & Lower-body strength & Box squat & Maximal \\
Session Two & Lower-body power & Jump squat & $-28 \%,-15 \%$ 1RM box squat \\
Session Three & Lower-body power & Jump squat & $0 \%, 20 \%, 30 \%$ 1RM box squat \\
Session Four & Lower-body power & Jump Squat & $40 \%, 50 \%, 60 \%$ 1RM box squat \\
\hline
\end{tabular}

RM, Repetition maximum. Twenty four hours separated each session. 